Methods This study was conducted among primary school children (6-10 years old) in the middle region of Iraq (five governorates). The total sample composed of 4,089 children randomly selected. The assessment of nutritional status was performed using height for age indicator for chronic malnutrition (stunted), the values were compared with the international reference values defined by the National Center for Health Statistics (NCHS) and classify them into mild, moderate, and sever stunted. The nutrient analysis was done through the dietary assessment using semi-quantitative food frequency questionnaires the assessment was converted to nutrient data (according to nutrient composition of Iraqi food) which was performed using software program especially designed for this study. Diagnosis and recording of dental caries was conducted according to World Health Organization criteria in 1997 using dmfs and DMFs indices for primary and permanent teeth respectively. Statistical analyses were performed using Analysis of Variance and Least Significant Different tests.

Results The prevalence of stunted, was found to be $49 \%$, where the mild grade was the most prevalent (29\%). The data presented in the study showed that there is a significant difference in mean of caries experience between different grades of nutritional status $(\mathrm{P}<0.01)$. The well-nourished children had significantly lower $\mathrm{dmfs}$ values $(11.76 \pm 0.95)$ than mild $(10.44 \pm 0.61)$ and sever stunted (16.32 \pm 1.07$)$. Concerning caries experience of permanent dentition, apposite figure were found, well-nourished children had higher DMFS values $(2.11 \pm 0.93)$ than mild $(1.39 \pm 0.07)$, moderate $(1.56 \pm 0.10)$, and sever stunted (1.14 \pm 0.18$)$. However all these differences were statistically not significant $(\mathrm{P}>0.05)$

The daily nutrients intake for the well-nourished children were recorded to be for protein $52.0 \pm 0.62$ grams, calcium $0.63 \pm 0.01$ grams, phosphorus $1.01 \pm 0.01$ grams, calcium/phosphate ratio $0.61 \pm 0.004$, iron $0.01 \pm 0.0002$ milligrams, vitamin A $6981 \pm 84.04$ I.U and vitamin C $0.10 \pm 0.002$ milligrams. These amounts tended to be decreased significantly with increased severity of malnutrition. Multiple linear regressions showed correlation between nutrients and dmfs, DMFs with $\mathrm{R}^{2}$ value $0.7 \%, 0.5 \%$ respectively.

Conclusion The oral cavity is considered a mirror of nutritional status of the body. Stunted children with inadequate nutrients intake during growth and development had a significant effect on tooth development and subsequent caries initiation and progression.

\section{PS25 DISCONTINUATION OF ANTIEPILEPTIC DRUGS IN PREGNANCY: A UK POPULATION BASED STUDY IN THE HEALTH IMPROVEMENT NETWORK (THIN)}

doi:10.1136/jech-2012-201753.124

'S Man, II Petersen, '2M Thompson, II Nazareth. 'Primary Care \& Population Health, UCL, London, UK; ${ }^{2}$ Cegedim Strategic Data Medical Research UK, Cegedim Strategic Data, London, UK

Background Since the thalidomide tragedy in the 1960s, many women are concerned with the use of drugs in pregnancy. Antiepileptic drugs (AEDs) are commonly used to treat epilepsy and bipolar disorders in women of child bearing age. However, some AEDs are associated with an increased risk of major congenital malformations. Untreated epilepsy and bipolar disorders in pregnancy may lead to devastating consequences for both mother and child. This leaves women and health care professionals with a dilemma as whether to continue AED medication in pregnancy. Using data from THIN, a UK primary care database, we examined pregnancy as a determinant for ceasing AED treatment.

Methods A cohort study of pregnant women receiving AEDs in UK primary care was conducted. We identified women who were prescribed AEDs in the three months before pregnancy. Time to last consecutive AED prescription in pregnancy was estimated whereby discontinuation of therapy was defined by a gap of more than three months between prescriptions. We identified a control group of twice as many non-pregnant women receiving AEDs. These women were randomly selected, but stratified within five year age bands and matched on indication for AEDs as for the pregnant women Cox's regression was used to compare the likelihood of discontinuing AEDs between pregnant and non-pregnant women.

Results In total, 934 women were prescribed AEDs three months before pregnancy. Pregnant women with epilepsy were twice as likely to cease AEDs compared to non-pregnant women (Hazard Ratio (HR):2.00, 95\% confidence interval (CI:1.62-2.47). Of 745 women with epilepsy, 601 (80.7\%) continued treatment into pregnancy and $465(62.4 \%)$ to the end of the second trimester. Of 1,490 non-pregnant women with epilepsy, 1,242 (83.4\%) and 1,071 (71.9\%) continued for comparable time periods.

Pregnant women with bipolar disorder or depression were three times as likely to cease AEDs compared to non-pregnant women (HR:3.07, 95\% CI:2.04-4.62). Of 54 pregnant women with bipolar disorder, $27(50.0 \%)$ continued into pregnancy, and only $8(14.8 \%)$ to the end of the second trimester. In 108 non-pregnant women with bipolar disorder, 82 (75.9\%) and 58 (53.7\%) continued for comparable periods.

Conclusion Pregnancy is a determinant for the discontinuation of AEDs during pregnancy, especially in women with bipolar disorder or depression despite the potentially severe consequences associated with not treating the underlying illness in pregnancy.

\section{PS26 PREVALENCE AND ASSOCIATIONS OF LIMITED HEALTH LITERACY IN CHRONIC KIDNEY DISEASE: A SYSTEMATIC REVIEW}

doi:10.1136/jech-2012-201753.125

${ }^{1}$ SDS Fraser, 'PJ Roderick, 'M Casey, ${ }^{2} \mathrm{MW}$ Taal, 'HM Yuen, ${ }^{3} \mathrm{D}$ Nutbeam. ${ }^{1}$ Public Health Sciences and Medical Statistics, University of Southampton, Southampton, UK; ${ }^{2}$ Renal Medicine, Royal Derby Hospital, Derby, UK; ${ }^{3}$ Vice Chancellor, University of Southampton, Southampton, UK

Background Chronic kidney disease (CKD) is a prevalent and growing problem, strongly associated with obesity, diabetes, hypertension, and cardiovascular disease. Health inequalities are recognised throughout the CKD pathway, including prevalence of risk factors, prevalence of $\mathrm{CKD}$, progression, and renal replacement therapy. There is evidence that an adequate level of health literacy (defined as the cognitive and social skills which determine the motivation and ability of individuals to gain access to, understand, and use information in ways that promote and maintain good health') contributes to improved disease management, and that inadequate health literacy is a potentially modifiable determinant of poor health outcomes and health inequalities in people with chronic disease. The aim of this review was to synthesise and critically appraise the literature evidence on the prevalence and associations of limited health literacy in CKD.

Methods Seven databases were searched using terms for CKD and health literacy $(\mathrm{HL})$. Cross sectional studies, and baseline data from cohort and randomised controlled trials were included where they assessed and presented the prevalence of limited HL using a validated tool in adults with CKD of any stage. The primary outcome was an objectively measured prevalence of limited HL in a population with CKD. The secondary outcome was associations of limited HL. Study quality was assessed by two reviewers using standardised criteria. Prevalence values were combined using a random effect model to give overall prevalence.

Results 82 studies were identified from searching, of which six met the inclusion criteria. The total number of people with CKD in all studies was 1,405. Five studies were in dialysis or transplant populations, and all were from the US. There was significant heterogeneity in the prevalence of limited HL $(9 \%$ to $32 \%$ (median $25 \%$, inter-quartile range $16 \%)$ ). The pooled prevalence of limited 
HL in all studies was $22.7 \%$ (95\% CI 20.6\%, 24.8\%). The review identified associations between limited HL and socioeconomic factors (lower education attainment, lower income), and certain process and outcome measures (lower likelihood of referral for transplant, higher mortality). Overall study quality was poor, with particular weaknesses of sampling and non-response.

Conclusion Limited health literacy is common among people with $\mathrm{CKD}$ and independently associated with socioeconomic factors and health outcomes. It may represent an important determinant of inequality of outcomes in CKD. There is a need for further investigation of limited health literacy in people with pre-end stage CKD, and in UK CKD populations.

\section{PS27 SERUM BILIRUBIN AND RISK OF CARDIOVASCULAR EVENTS AND DEATH IN A STATIN-TREATED POPULATION: A COHORT STUDY}

doi:10.1136/jech-2012-201753.126

LJ Horsfall, I Petersen, I Nazareth. Primary Care and Population Health, UCL, London, UK

Background Serum bilirubin is an endogenous antioxidant with a strong genetic component that may be a marker for future cardiovascular disease (CVD) risk. We examined the relationship between serum bilirubin levels recorded prior to statin prescription, primarily as test for liver function, and the diagnosis or death from CVD. We also examined whether bilirubin levels increased following statin prescription independently of liver enzymes, which has been shown in animal models and could contribute to the purported health benefits of statins in addition to cholesterol reduction.

Methods All patients with liver function tests three months prior to first statin treatment between January 1st 2000 and December 31 st 2010 and no history of liver disease or CVD were extracted from The Health Improvement Network (THIN) primary care database. Restricted cubic spline Poisson regressions were fitted on bilirubin levels and adjusted for traditional cardiovascular risk factors to estimate incidence rate ratios.

Results In total 130,052 patients met the inclusion criteria and after a median follow-up of 43 months, there were 5,938 coronary heart disease (CHD) events, 2,438 stroke events, and 5,185 deaths from any cause. In men, the incidence of CHD in the lowest decile category of bilirubin $(1-6 \mu \mathrm{mol} / \mathrm{L})$ was 176 per 10,000 person years (PYs) compared with 139 per 10,000 PYs in the highest decile (19-40 $\mu \mathrm{mol} / \mathrm{L})$. The result for stroke was 72 versus 52 per 10,000 PYs and for death 139 versus 92 per 10,000 PYs. Similar differences were seen for women. The adjusted associations with bilirubin were L-shaped with a negative relationship up to around $10-15 \mu \mathrm{mol} / \mathrm{L}$. The models predicted that, compared to patients with the median bilirubin level $(10 \mu \mathrm{mol} / \mathrm{L})$, those with a similar CVD risk profile but a bilirubin level of $5 \mu \mathrm{mol} / \mathrm{L}$ had a $19 \%$ (95\% CI: $11-27 \%$ ) higher rates of CHD, a $22 \%$ (95\% CI: $10-36 \%$ ) higher rates of stroke, and a $26 \%$ (95\% CI: $17-35 \%$ ) higher rate of death. A dose-dependent increase in mean bilirubin level was seen following atorvastatin prescription but not for simvastatin.

Conclusion Low bilirubin prior to statin prescription is an independent risk factor for CVD and death. Further work is needed to examine whether pleotrpic effects of statins can be explained by alterations in bilirubin production and/or elimination.

\section{PS28 IMPACT OF NUMBER AND TYPE OF COMORBIDITY ON DEPRESSION PREVALENCE AND HEALTH CARE COSTS. POPULATION-BASED COHORT STUDY}

doi:10.1136/jech-2012-201753.127

${ }^{1} \mathrm{~N}$ Bhattarai, ${ }^{1} \mathrm{~J}$ Charlton, ${ }^{2} \mathrm{C}$ Rudisill, 'M C Gulliford. 'Department of Primary Care and Public Health Sciences, King's College London, London, UK; ${ }^{2}$ Department of Social Policy and LSE Health, London School of Economics, London, UK
Background The degree to which the number of comorbidities and the presence of depression impact health care use and costs has been unexplored employing actual resource use data representing a general population. We aimed to evaluate the impact of number and type of comorbidities on depression prevalence, health care utilisation and health care costs.

Methods Cohort study and included in the study were 300,020 participants aged 30 years of age registered with the UK General Practice Research Database. We used the UK General Practice Research Database to examine participants' diagnoses and resource use in primary and secondary care from 2005 to 2009. Healthcare unit costs and the costs of each individual prescription item were analysed.

Results In participants with no comorbidity, the age-standardised prevalence of depression was $7 \%$ in men and $14 \%$ in women. The likelihood of having depression increased in participants with single comorbidities including diabetes mellitus (men 13\%, women $22 \%$ ), CHD (men $15 \%$, women $24 \%$ ), stroke (men $14 \%$, women $26 \%$ ) or colorectal cancer (men 10\%, women 21\%). Patients with concurrent diabetes, CHD and stroke had a very high prevalence of depression (men 23\%, women 49\%) with women being more likely to suffer depression. Patients with a single comorbidity were 1.63 (95\% confidence interval 1.59 to 1.66) times more likely to be depressed than those without comorbidity while those with two and three comorbidities were 1.96 (1.89 to 2.03) and 2.35 (2.03 to 2.59) times more likely. Depression increased the total costs of resources utilised per year in all participants across both genders, all age groups and across all comorbidities analysed in this study. Individuals with depression had higher total annual health care costs (males $=£ 1014$, females=£1212) than those without comorbidity or depression (males $=£ 380$, females $=£ 517$ ). Those with diabetes alone had $£ 1144$ for males and $£ 1393$ for females but $£ 2534$ for males and $£$ 3017 for females when depression was present alongside diabetes. When patients had diabetes, CHD and had suffered a stroke these patients' costs were $£ 1541$ for males and $£ 1879$ for females without depression and $£ 3420$ for males and $£ 4072$ for females with depression. Depression increased the associated cost of any comorbidity.

Conclusion The prevalence of depression appears to be more strongly determined by the number of comorbidities rather than the precise nature of the comorbid diagnoses. Additional costs of health care utilisation are considerably higher when depression is associated with single or multiple comorbidities.

\section{PS29 YOUTH EXPOSURE TO ONLINE ALCOHOL ADVERTISING IN THE UK: AN ANALYSIS OF SOCIAL MEDIA WEBSITES}

doi:10.1136/jech-2012-201753.128

'E Winpenny, ${ }^{2} \mathrm{TM}$ Marteau, ${ }^{1} \mathrm{E}$ Nolte. ${ }^{1}$ Health $\&$ Healthcare, RAND Europe, Cambridge, UK; ${ }^{2}$ Behaviour and Health Research Unit, University of Cambridge, Cambridge, UK

Background There is increasing evidence that youth exposure to alcohol marketing is a risk factor for underage drinking. In 2011, online marketing became the largest channel for marketing for the first time, overtaking television. However, there is little understanding of the level of exposure of young people to online alcohol marketing.

Methods We obtained data on the top 3 social media sites in the UK for each month from December 2010 to May 2011, based on unique user figures, by gender and age (6-14, 15-24). We analysed the reach (the proportion of available internet users who used the site in each month) and impressions (the number of individual pages viewed on the site in each month) of the overall top three social media sites, Facebook, YouTube and Twitter in each demographic. Using data from the top 10 TV channels in the UK we identified 5 drinks brands, which had the highest TV advertising exposure to children (4-15) during this 6 month period. During February and March 2012, we examined each of these brands across the 